\title{
Existence and Ulam Stability of Solution for Some Backward Impulsive Differential Equations on Banach Spaces
}

\begin{abstract}
A. Mahmoudi and A. Kessi
Abstract. In this paper, we study the existence and the Ulam stability of a solution to nonlinear backward impulsive differential equations with local or nonlocal conditions in Banach spaces. Using well-known classical fixed point theorems, we prove the existence of a solution. Subsequently, we prove the generalized Ulam-Hyers-Rassias stability of the solution to the problem. The obtained results are illustrated by some examples.
\end{abstract}

Key Words: Backward impulsive differential equations, fixed point, Ulam stability

Mathematics Subject Classification 2010: 34A12, 34A37, 34D20, 34K45

\section{Introduction}

The theory of impulsive differential equations is an adequate mathematical model for describing processes that experience a sudden change of their state at certain moments between intervals of continuous evolution. Because the duration of these changes is often negligible compared to the total extent of the process, they can be reasonably well approximated as being instantaneous changes of state, or in the form of impulses. Thus, such processes tend to be more suitably modelled by impulsive differential equations, which allow for discontinuities in the evolution of the state.

Processes of the described type often arise naturally, for example, in physics, chemical technology, population dynamics, aeronautics, biotechnology, chemotherapy, optimal control, ecology, economics, and engineering. The corresponding theory has seen significant development over the past decades. For more details, we refer the interested reader to the monographs of Lakshmikantham et al. [15] and Samoilenko and Perestyuk [23] for the 
case of ordinary impulsive system, works [12, 13, 21, 22] for partial differential and partial functional differential equations with impulses and the references therein.

Many authors were interested in the solvability of boundary value problems with impulses. Nieto [19] studied the existence of solutions to the first order periodic problem with impulse. Chen, Tisdell and Yuan [8] considered the solvability of the periodic problem with impulse. Lin and Jiang [16] studied the existence of positive solutions for the second order Dirichlet boundary value problem with impulse.

Usually, the initial conditions for the differential equations are given in a forward manner, that is, starting at $t=0$. But for some classes of problems in which the initial state set is unknown the procedure may be more convenient if one considers backward initial conditions, i.e., at $t=T$. This approach plays a vital role in many physical areas. A typical example of such a problem is the backward heat problem (BHP), also known as the final value problem. For application in stochastic differential equations, see, for example, [10].

However, as far as we know, there are no results on the existence and stability of solutions to backward impulsive differential equations. In this paper, using some well known classical fixed point theorems, we study the problem of the existence of solutions and their Ulam stability for the following backward differential equations in Banach spaces

$$
\begin{cases}u^{\prime}(t)=f(t, u(t)), & t \in J=[0, T], t \neq t_{k}, \\ \left.\Delta u\right|_{t=t_{k}}=I_{k}\left(u\left(t_{k}^{-}\right)\right), & k=1, \ldots, m, \\ u(T)=u_{T} & \end{cases}
$$

where $0=t_{0}<t_{1}<t_{2}<\ldots<t_{m}<t_{m+1}=T,\left.\Delta u\right|_{t=t_{k}}=u\left(t_{k}^{+}\right)-u\left(t_{k}^{-}\right)$ represents the jump of the function $u$ at $t_{k}, I_{k}: \mathbb{R} \rightarrow \mathbb{R}, k=1,2, \ldots, n$, are appropriate functions, and $f: J \times \mathbb{R} \longrightarrow \mathbb{R}$ is a nonlinear real function. Our method of study is to convert the initial value problem (1) into an equivalent integral equation and apply Schaefer, Banach or Krasnoselskii fixed point theorem. Further, we prove the existence of a unique solution or at least one solution to this problem with local and nonlocal conditions. Consider the following nonlocal problem

$$
\begin{cases}u^{\prime}(t)=f(t, u(t)), & t \in J=[0, T], t \neq t_{k}, k=1, \ldots, m \\ \left.\Delta u\right|_{t=t_{k}}=I_{k}\left(u\left(t_{k}^{-}\right)\right), & k=1, \ldots, m \\ u(T)-g(u)=u_{T}, & \end{cases}
$$

where $f$ and $I_{k}, k=1, \ldots, m$, are defined as in the previous paragraph and $g: P C(J, \mathbb{R}) \rightarrow \mathbb{R}$ is a continuous function. Nonlocal conditions were first investigated by Byszewski and Lakshmikantham [7]. Using the Banach fixed point theorem, they obtained conditions for the existence and uniqueness of 
mild solutions to nonlocal differential equations. Byszewski [6] proved the existence and uniqueness of mild and classical solutions of nonlocal Cauchy problem.

The nonlocal problem was motivated by physical problems. Also, it was demonstrated that the nonlocal problems have better effects in applications than the classical Cauchy problems. Let us mention, for example, nonlocal neural networks, nonlocal pharmacokinetics, nonlocal pollution, and nonlocal combustion. Particularly, in 1999, Byszewski [5] obtained conditions for the existence and uniqueness of classical solution to a class of abstract functional differential equations with nonlocal conditions of the form

$$
\left\{\begin{array}{l}
u^{\prime}(t)=f(t, u(t), u(a(t))), \quad t \in I, \\
u\left(t_{0}\right)+\sum_{k=1}^{p} c_{k} u\left(t_{k}\right)=x_{0},
\end{array}\right.
$$

where $I:=\left[t_{0}, t_{0}+T\right], t_{0}<t_{1}<\ldots<t_{p} \leq t_{0}+T, T>0 ; f: I \times E^{2} \rightarrow E$ and $a: I \rightarrow I$ are given functions; $E$ is a Banach space, $x_{0} \in E, c_{k} \neq 0, k=$ $1,2, \ldots, p$, and $p \in \mathbb{N}$. The author pointed out that if $c_{k} \neq 0, k=1,2, \ldots, p$, then the results of the paper can be applied in kinematics to determine the evolution $t \rightarrow u(t)$ of a location of a physical object, for which we do not know the positions $u(0), u\left(t_{1}\right), \ldots, u\left(t_{p}\right)$, but we know that the nonlocal condition holds. To check the Ulam stability, we proceed as Wang et all [24].

\section{Preliminaries}

By $C(J, \mathbb{R})$ we denote the Banach space of all continuous functions from $J=[0, T]$ into $\mathbb{R}$ endowed with the norm

$$
\|u\|_{\infty}=\sup _{t \in J}|u(t)|
$$

The set of piecewise continuous functions

$$
\begin{aligned}
P C(J, \mathbb{R})= & \left\{u: J \rightarrow \mathbb{R}:\left.u\right|_{\left(t_{k}, t_{k+1}\right)} \in C\left(\left(t_{k}, t_{k+1}\right), \mathbb{R}\right), k=0,1, \ldots, m,\right. \\
& \left.u\left(t_{k}^{-}\right)=u\left(t_{k}\right) \text { and } u\left(t_{k}^{+}\right) \text {exists }\right\}
\end{aligned}
$$

is a Banach space with the norm

$$
\|u\|_{P C}=\sup _{t \in J}|u(t)| .
$$

Define the set $B_{r}=\{u \in P C(J, \mathbb{R}):\|u\| \leq r\}$.

Below we formulate some known results. We start with Ascoli-Arzela and PC-type Ascoli-Arzela theorem [25] and then formulate fixed point theorems, respectively, by Banach, Schafer and Krasnoselskii. 
Theorem 1 Let $A$ be a family of fonctions in $C[K]$, where $K$ is compact. Then $A$ is compact if and only if $A$ is closed, bounded, and equicontinuous.

Theorem 2 Let $E$ be a Banach space and $W \subset P C(J, E)$ be such that

(i) $W$ is uniformly bounded subset of $P C(J, E)$;

(ii) $W$ is equicontinuous in $\left(t_{k}, t_{k+1}\right), k=0,1, \ldots, m$, where $t_{0}=0$ and $t_{m+1}=T$

(iii) $W(t)=\left\{u(t) \mid u \in W ; t \in J \backslash\left\{t_{1}, \ldots, t_{m}\right\}\right\}, W\left(t_{k}^{+}\right)=\left\{u\left(t_{k}^{+}\right) \mid u \in W\right\}$ and $W\left(t_{k}^{-}\right)=\left\{u\left(t_{k}^{-}\right) \mid u \in W\right\}$ are relatively compact subsets of $E$.

Then $W$ is a relatively compact subset of $P C(J, E)$.

Theorem 3 A contractive mapping on a Banach space has a unique fixed point.

Theorem 4 Let $E$ be a Banach space and $U \subset E$ a convex set such that $0 \in U$. Let $T$ be an operator defined on $E$ such that $T: U \rightarrow U$ is completely continuous. If

$$
\Omega=\{u \in U: u=\lambda T u, \lambda \in] 0,1[\}
$$

is bounded, then $T$ admits at least one fixed point in $E$.

Theorem 5 Let $S$ be a closed convex nonempty subset of a Banach space $E$. Let $P$ and $Q$ be two operators satisfying the following conditions:

1. $P x+Q y \in S$ whenever $x, y \in S$;

2. $P$ is a contraction mapping;

3. $Q$ is compact and continuous.

Then there exist $z \in S$ such that $z=P z+Q z$, i.e., the operator $P+Q$ admits a fixed point on $S$.

The following generalized Gronwall inequality for piecewise continuous functions will be widely used when studying the Ulam stability.

Lemma 1 (Gronwall inequality [2]) Let for $t \geq t_{0} \geq 0$, the following inequality holds

$$
x(t) \leq a(t)+b \int_{t_{0}}^{t} x(s) d s+\sum_{t_{0}<t_{k}<t} \beta_{k} x\left(t_{k}\right),
$$

where $x, a \in P C\left(\left[t_{0}, \infty\right), \mathbb{R}^{+}\right)$, $a$ is nondecreasing and $b, \beta_{k}>0$. Then for $t \geq t_{0}$, the following inequality is valid:

$$
x(t) \leq a(t) \prod_{t_{0}<t_{k}<t}\left(1+\beta_{k}\right) \exp \left(\int_{t_{0}}^{t} b(s) d s\right) .
$$

For more integral inequalities of Gronwall type for piecewise continuous functions, see [1, 2]. 


\section{Existence results}

In this section, our attention is focused on the main results on the existence of a solution to the problem (1). We discuss conditions under which this problem has exactly one solution or at least one solution. tions:

In the study of the problem (1), we will work with the following assump-

(H1) The function $f: J \times \mathbb{R} \longrightarrow \mathbb{R}$ is continuous.

(H2) There exists a positive constant $\lambda$ such that for any $t \in[0, T]$ and $x, y \in \mathbb{R},|f(t, x)-f(t, y)| \leq \lambda|x-y|$.

(H3) There exists a positive constant $\theta$ such that $|f(t, x)|<\theta$ for any $t \in[0, T]$ and $x \in \mathbb{R}$.

(H4) $|f(t, x)| \leq r$ for any $t \in[0, T]$ and $x \in B_{r}, r \in \mathbb{R}_{+}$.

(H5) There exists a constant $\mu>0$, such that $\left|I_{k}(x)-I_{k}(y)\right| \leq \mu|x-y|$ for any $x, y \in \mathbb{R}, k=1, \ldots, m$.

(H6) The functions $I_{k}: \mathbb{R} \rightarrow \mathbb{R}$ are continuous and there exists a positive constant $\gamma$ such that $\left|I_{k}(x)\right|<\gamma$ for any $x \in \mathbb{R}, k=1, \ldots, m$.

A function $u \in P C(J, \mathbb{R})$ will be called a solution to (1) if its derivative exists on $J^{\prime}=J-\left\{t_{k}, k=1,2,3, \ldots, n\right\}$ and $u$ satisfies the equation

$$
u^{\prime}(t)=f(t, u(t)), \quad t \in J^{\prime},
$$

and the conditions

$$
\begin{aligned}
& \left.\Delta u\right|_{t=t_{k}}=I_{k}\left(u\left(t_{k}^{-}\right)\right), \quad k=1, \ldots, m, \\
& u(T)=u_{T}
\end{aligned}
$$

Lemma 2 A function $u$ is a solution to the integral equation

$$
u(t)=u_{T}-\sum_{p=0}^{k-1} I_{m-p}\left(u\left(t_{m-p}^{-}\right)\right)-\int_{t}^{T} h(s) d s
$$

for $t \in\left(t_{m-k}, t_{m-k+1}\right), k=0, \ldots, m$, if and only if $u$ is a solution to the backward impulsive equation

$$
\left\{\begin{array}{l}
u^{\prime}(t)=h(t), \quad t \in J=[0, T], \quad t \neq t_{k}, \\
\left.\Delta u\right|_{t=t_{k}}=I_{k}\left(u\left(t_{k}^{-}\right)\right), \\
u(T)=u_{T}
\end{array}\right.
$$


Proof. Assume $u$ satisfies (4). Then for $t \in\left(t_{m}, T\right)$, we have

$$
u(t)=u_{T}-\int_{t_{m}}^{T} h(s) d s+\int_{t_{m}}^{t} h(s) d s .
$$

We will proceed by induction on $m$. For $t \in\left(t_{m-1}, t_{m}\right)$, we can write

$$
\begin{aligned}
u(t) & =u\left(t_{m}^{-}\right)-\int_{t_{m-1}}^{t_{m}} h(s) d s+\int_{t_{m-1}}^{t} h(s) d s \\
& =-\triangle\left(u\left(t_{m}\right)\right)+u\left(t_{m}^{+}\right)-\int_{t_{m-1}}^{t_{m}} h(s) d s+\int_{t_{m-1}}^{t} h(s) d s \\
& =-I_{m}\left(u\left(t_{m}^{-}\right)\right)+u_{T}-\int_{t_{m}}^{T} h(s) d s-\int_{t_{m-1}}^{t_{m}} h(s) d s+\int_{t_{m-1}}^{t} h(s) d s .
\end{aligned}
$$

Further, for any $k=0,1, \ldots, m$ and $t \in\left(t_{m-k}, t_{m-k+1}\right)$, we obtain

$$
\begin{aligned}
u(t) & =u_{T}-\sum_{p=0}^{k-1} I_{m-p}\left(u\left(t_{m-p}^{-}\right)\right)-\sum_{p=0}^{k} \int_{t_{m-p}}^{t_{m-p+1}} h(s) d s+\int_{t_{m-k}}^{t} h(s) d s \\
& =u_{T}-\sum_{p=0}^{k-1} I_{m-p}\left(u\left(t_{m-p}^{-}\right)\right)-\int_{t_{m-k}}^{T} h(s) d s+\int_{t_{m-k}}^{t} h(s) d s \\
& =u_{T}-\sum_{p=0}^{k-1} I_{m-p}\left(u\left(t_{m-p}^{-}\right)\right)-\int_{t}^{T} h(s) d s .
\end{aligned}
$$

Conversely, assume that $u$ satisfies the impulsive integral equation (3). If $t \in\left(t_{m}, T\right)$, then $u(T)=u_{T}$.

If $t \in\left(t_{m-k}, t_{m-k+1}\right), k=0, \ldots, m$, by differentiation (3), we get

$$
u^{\prime}(t)=h(t) .
$$

It remains to note that

$$
\left.\Delta u\right|_{t=t_{k}}=I_{k}\left(u\left(t_{k}^{-}\right)\right), \quad k=1, \ldots, m .
$$

First, we discuss conditions under which the problem (1) has a unique solution. The following result is based on the Banach fixed point theorem.

Theorem 6 Assume that the conditions $(\mathbf{H 1}),(\mathbf{H} 2)$ and $(\mathbf{H 5})$ are verified and

$$
m \mu+\lambda T<1 .
$$

Then the problem (1) has a unique solution in $P C(J, \mathbb{R})$. 
Proof. We transform the problem (1) into a fixed point problem. Consider the operator $F: P C(J, \mathbb{R}) \rightarrow P C(J, \mathbb{R})$ defined by

$$
F(u)(t)=u_{T}-\sum_{t<t_{k}<T} I_{k}\left(u\left(t_{k}^{-}\right)\right)-\int_{t}^{T} f(s, u(s)) d s .
$$

Clearly, if the operator $F$ has a fixed point, then it is a solution to the problem (1).

Let $u, v \in P C(J, \mathbb{R})$. Then for each $t \in J$, we have

$$
\begin{aligned}
& |F(u)(t)-F(v)(t)| \\
& \quad \leq \sum_{t<t_{k}<T}\left|I_{k}\left(u\left(t_{k}^{-}\right)\right)-I_{k}\left(v\left(t_{k}^{-}\right)\right)\right| \int_{t}^{T}|f(s, u(s))-f(s, v(s))| d s \\
& \quad \leq \mu \sum_{t<t_{k}<T}\left|u\left(t_{m-p}^{-}\right)-v\left(t_{m-p}^{-}\right)\right|+\lambda \int_{t}^{T}|u(s)-v(s)| d s \\
& \quad \leq m \mu|u(t)-v(t)|+\lambda T|u(t)-v(t)| \\
& \quad=(m \mu+\lambda T)|u(t)-v(t)| .
\end{aligned}
$$

Hence, by (5), $F$ is a contraction. Then, by the Banach contraction principle, $F$ has a unique fixed point, which is a solution to the problem (1).

The following result provides sufficient conditions for the existence of at least one solution to the problem (1). It is based on the Schaefer's fixed point theorem.

Theorem 7 If the conditions $(\mathbf{H 1}),(\mathbf{H} 2)$ and $(\mathbf{H} 4)-(\mathbf{H 6})$ are satisfied, then the problem (1) has at least one solution in $P C(J, \mathbb{R})$.

Proof. For the sake of convenience, the proof of this result is divided into four steps.

Step 1: The operator $F$ is continuous. Let $\left(u_{n}\right)$ be such a sequence that $u_{n} \rightarrow u$ on $J$. Then, for all $t \in[0, T]$,

$$
\begin{aligned}
& \left|F\left(u_{n}\right)(t)-F(u)(t)\right| \\
& \quad \leq \sum_{t<t_{k}<T}\left|I_{k}\left(u_{n}\left(t_{k}^{-}\right)\right)-I_{k}\left(u\left(t_{k}^{-}\right)\right)\right|+\int_{t}^{T}\left|f\left(s, u_{n}(s)\right)-f(s, u(s))\right| d s .
\end{aligned}
$$

Since $f$ and $I_{k}, k=1, \ldots, m$, are continuous functions, we have

$$
\left\|F u_{n}-F u\right\|_{\infty} \rightarrow 0 \text { as } n \rightarrow \infty,
$$

which implies that $F$ is continuous. 
Step 2: $F$ maps bounded sets into bounded sets in $P C(J, \mathbb{R})$. For all $u \in B_{r}$, we have

$$
\begin{aligned}
|F u(t)| & =\left|u_{T}-\sum_{t<t_{k}<T} I_{k}\left(u\left(t_{k}^{-}\right)\right)-\int_{t}^{T} f(s, u(s)) d s\right| \\
& \leq\left|u_{T}\right|+\left|\sum_{t<t_{k}<T} I_{k}\left(u\left(t_{k}^{-}\right)\right)\right|+\left|\int_{t}^{T} f(s, u(s)) d s\right| \\
& \leq\left|u_{T}\right|+\sum_{t<t_{k}<T}\left|I_{k}\left(u\left(t_{k}^{-}\right)\right)\right|+\int_{t}^{T}|f(s, u(s))| d s \\
& \leq\left|u_{T}\right|+\sum_{t<t_{k}<T}\left|I_{k}\left(u\left(t_{k}^{-}\right)\right)\right|+r \int_{t}^{T} d s \\
& \leq\left|u_{T}\right|+m \gamma+r T=\rho .
\end{aligned}
$$

Hence, the operator $F$ maps the bounded set $B_{r}$ into the bounded set $B_{\rho}$.

Step 3: $F$ maps bounded sets into the equicontinuous sets of $P C(J, \mathbb{R})$. Let $\tau_{1}, \tau_{2} \in[0, T], t_{k}<\tau_{1}<\tau_{2}<t_{k+1}, k=0,1, \ldots, m-1$, and let $u \in B_{r}$. Then

$$
\begin{aligned}
\mid F(u) & \left(\tau_{2}\right)-F(u)\left(\tau_{1}\right) \mid \\
& \leq \sum_{\tau_{1}<t_{k}<\tau_{2}}\left|I_{k}\left(u\left(t_{k}^{-}\right)\right)\right|+\int_{\tau_{1}}^{\tau_{2}}|f(s, u(s))| d s=\int_{\tau_{1}}^{\tau_{2}}|f(s, u(s))| d s .
\end{aligned}
$$

The right-hand side of this inequality tends to zero when $\tau_{1}$ tends to $\tau_{2}$. By the precedent steps, together with the Ascoli-Arzela theorem, we conclude that $F$ is equicontinuous on interval $\left[t_{k}, t_{k+1}\right]$.

Thus, by the PC-type Arzela-Ascoli theorem, we conclude that $F: B_{r} \rightarrow$ $B_{\rho}$ is continuous and completely continuous.

Step 4: The set $\Omega=\{u \in P C(J, \mathbb{R}): u=\lambda F(u), 0<\lambda<1\}$ is bounded. Since for any $u \in \Omega$, we have $u=\lambda F(u)$ for some $0<\lambda<1$, for all $t \in[0, T]$, we can write

$$
\begin{aligned}
|u(t)| & =\lambda\left|u_{T}-\sum_{t<t_{k}<T} I_{k}\left(u\left(t_{k}^{-}\right)\right)-\int_{t}^{T} f(s, u(s)) d s\right| \\
& \leq\left|u_{T}\right|+\left|\sum_{t<t_{k}<T} I_{k}\left(u\left(t_{k}^{-}\right)\right)\right|+\int_{t}^{T}|f(s, u(s))| d s \\
& \leq\left|u_{T}\right|+\sum_{t<t_{k}<T}\left|I_{k}\left(u\left(t_{k}^{-}\right)\right)\right|+|f(t, u(t))| \int_{t}^{T} d s \\
& \leq\left|u_{T}\right|+m \gamma+\theta T .
\end{aligned}
$$

This proves that $\Omega$ is bounded. Hence, by the Schaefer's fixed point theorem, $F$ has a fixed point which is a solution to the problem (1). 


\section{Nonlocal Backward Impulsive Differential Equations}

In this section, we generalize the results of the previous section to nonlocal impulsive differential equations (2).

Let us introduce the following assumptions:

(H7) There exists a positive constant $C$ such that $|g(x)-g(y)| \leq C\|x-y\|$ for any $x, y \in P C(J, \mathbb{R})$.

(H8) There exists a positive constant $\kappa$ such that $|g(u)| \leq \kappa$ for any function $u \in P C(J, \mathbb{R})$.

The equation (2) is equivalent to the following integral equation

$$
u(t)=u_{T}+g(u(t))-\sum_{t<t_{k}<T} I_{k}\left(u\left(t_{k}^{-}\right)\right)-\int_{t}^{T} f(s, u(s)) d s .
$$

Theorem 8 Assume that the conditions (H1), (H2) and (H5) are satisfied, and

$$
C+m \mu+\lambda T<1 \text {. }
$$

Then the problem (2) has a unique solution in $P C(J, \mathbb{R})$.

Proof. Consider the operator $F: P C(J, R) \rightarrow P C(J, R)$ defined by

$$
F(u)(t)=u_{T}+g(u(t))-\sum_{t<t_{k}<T} I_{k}\left(u\left(t_{k}^{-}\right)\right)-\int_{t}^{T} f(s, u(s)) d s .
$$

First, we show that $F$ is a contraction. Let $u, v \in P C(J, R)$. Then, for each $t \in J$, we have

$$
\begin{aligned}
\mid F(u)( & (t)-F(v)(t)|\leq| g(u(t))-g(v(t)) \mid \\
& +\sum_{t<t_{k}<T}\left|I_{k}\left(u\left(t_{k}^{-}\right)\right)-I_{k}\left(v\left(t_{k}^{-}\right)\right)\right|+\int_{t}^{T}|f(s, u(s))-f(s, v(s))| d s \\
\leq & C|u(t)-v(t)|+\mu \sum_{t<t_{k}<T}\left|u\left(t_{m-p}^{-}\right)-v\left(t_{m-p}^{-}\right)\right| \\
& +\lambda \int_{t}^{T}|u(s)-v(s)| d s \\
\leq & C|u(t)-v(t)|+m \mu|u(t)-v(t)|+\lambda T|u(t)-v(t)| \\
= & (C+m \mu+\lambda T)|u(t)-v(t)| .
\end{aligned}
$$

Hence, by (6), $F$ is a contraction. Then, by the Banach contraction principle, we deduce that $F$ has a unique fixed point which is a solution to the problem (2). 
Theorem 9 If (H1), (H3) and $(\mathbf{H 6})-(\mathbf{H} 8)$ are satisfied and $C<1$, then the problem (2) has at least one solution in $P C(J, \mathbb{R})$.

Proof. Put

$$
r \geq \frac{\left|u_{T}\right|+\kappa}{1-(m \gamma+\theta T)},
$$

and define the operators $P$ and $Q$ on the compact set $B_{r} \subset P C(J, \mathbb{R})$ by

$$
\begin{aligned}
P u(t) & =u_{T}+g(u(t)), \\
Q u(t) & =-\sum_{t<t_{k}<T} I_{k}\left(u\left(t_{k}^{-}\right)\right)-\int_{t}^{T} f(s, u(s)) d s .
\end{aligned}
$$

For all $u \in B_{r}$, we have

$|P u(t)|=\left|u_{T}+g u(t)\right| \leq\left|u_{T}\right|+|g u(t)| \leq\left|u_{T}\right|+\kappa \leq r(1-(m \gamma+\theta T)) \leq r$.

Hence, the operator $P$ maps $B_{r}$ into itself. Further, for all $u, v \in P C(J, \mathbb{R})$, we can write

$$
|P u(t)-P v(t)|=|g u(t)-g v(t)| \leq C|u(t)-v(t)|
$$

and hence, the operator $P$ satisfies the contraction property. Since

$$
\begin{aligned}
|Q v(t)| & \leq \sum_{t<t_{k}<T}\left|I_{k}\left(v\left(t_{k}^{-}\right)\right)\right|+\int_{t}^{T}|f(s, v(s))| d s \\
& \leq(m \gamma+\theta T)|v(t)|
\end{aligned}
$$

we can write

$$
\begin{aligned}
|P u(t)+Q v(t)| & \leq|P u(t)|+|Q v(t)| \\
& \leq\left|u_{T}\right|+\kappa+(\gamma m+\theta T)|v(t)| \\
& \leq\left|u_{T}\right|+\kappa+(m \gamma+\theta T) r \\
& \leq r .
\end{aligned}
$$

Therefore, if $u, v \in B_{r}$, then $P u+Q v \in B_{r}$. By (H1), $Q$ is continuous and by the inequality (7), it is uniformly bounded on $B_{r}$. The equicontinuity of $Q v(t)$ follows from Theorem 7. Hence, by the Arzela-Ascoli theorem, $Q\left(B_{r}\right)$ is relatively compact, which implies that $Q$ is compact. Therefore, using Krasnoselkii theorem, we conclude that there exists a solution to the equation (2). 


\section{Ulam stability for Some Nonlinear Back- ward Impulsive Differential Equations}

In this section, we study the Ulam stability of the solution to the problem (1).

Now, we introduce Ulam's type stability concepts for the equation (1). Let $\varepsilon>0, \psi \geq 0$ and $\varphi \in P C\left(J, \mathbb{R}^{+}\right)$is nondecreasing. Consider the following inequalities:

$$
\begin{aligned}
& \begin{cases}\left|y^{\prime}(t)-f(t, y(t))\right| \leq \varepsilon, & t \in J^{\prime}, \\
|\Delta y|_{t=t_{k}}-I_{k}\left(y\left(t_{k}^{-}\right)\right) \mid \leq \varepsilon, & k=1, \ldots, m,\end{cases} \\
& \begin{cases}\left|y^{\prime}(t)-f(t, y(t))\right| \leq \varphi(t), & t \in J^{\prime}, \\
|\Delta y|_{t=t_{k}}-I_{k}\left(y\left(t_{k}^{-}\right)\right) \mid \leq \psi, & k=1, \ldots, m,\end{cases}
\end{aligned}
$$

and

$$
\left\{\begin{array}{l}
\left|y^{\prime}(t)-f(t, y(t))\right| \leq \varepsilon \varphi(t), \quad t \in J^{\prime} \\
|\Delta y|_{t=t_{k}}-I_{k}\left(y\left(t_{k}^{-}\right)\right) \mid \leq \varepsilon \psi, \quad k=1, \ldots, m .
\end{array}\right.
$$

Definition 1 Equation (1) is Ulam-Hyers stable if there exists a real number $c_{f, m}>0$ such that for each $\varepsilon>0$ and for each solution $y \in P C^{1}(J, \mathbb{R})$ of the inequality (8), there exists a solution $u \in P C^{1}(J, \mathbb{R})$ of the equation (1) with

$$
|y(t)-u(t)| \leq c_{f, m} \varepsilon, \quad t \in J^{\prime} .
$$

Definition 2 Equation (1) is generalized Ulam-Hyers stable if there exists $\theta_{f, m} \in C\left(\mathbb{R}^{+}, \mathbb{R}^{+}\right), \theta_{f, m}(0)=0$ such that for each solution $y \in P C^{1}(J, \mathbb{R})$ of the inequality (8), there exists a solution $u \in P C^{1}(J, \mathbb{R})$ of the equation (1) with

$$
|y(t)-u(t)| \leq \theta_{f, m}(\varepsilon), \quad t \in J^{\prime}
$$

Definition 3 Equation (1) is Ulam-Hyers-Rassias stable with respect to $(\varphi, \psi)$ if there exists $c_{f, m, \varphi}>0$ such that for each $\varepsilon>0$ and for each solution $y \in P C^{1}(J, \mathbb{R})$ of the inequality $(10)$, there exists a solution $u \in P C^{1}(J, \mathbb{R})$ of the equation (1) with

$$
|y(t)-u(t)| \leq c_{f, m, \varphi} \varepsilon(\varphi(t)+\psi), \quad t \in J^{\prime} .
$$

Definition 4 Equation (1) is said to be generalized Ulam-Hyers-Rassias stable with respect to $(\varphi, \psi)$ if there exists $c_{f, m, \varphi}>0$ such that for each solution $y \in P C^{1}(J, \mathbb{R})$ of inequality $(9)$, there exists a solution $u \in P C^{1}(J, \mathbb{R})$ of equation (1) with

$$
|y(t)-u(t)| \leq c_{f, m, \varphi}(\varphi(t)+\psi), \quad t \in J^{\prime} .
$$

Remark 1 It is clear that (i) Definition 1 implies Definition 2: (ii) Definition 3 implies Definition 4; (iii) Definition 3 for $\varphi(t)=\psi=1$ implies Definition 1 . 
Proposition 1 A function $y \in P C^{1}(J, \mathbb{R})$ is a solution of inequality (8) if and only if there is $g \in P C(J, \mathbb{R})$ and a sequence $g_{k}, k=1,2, \ldots, m$ (which depends on $y$ ) such that

(i) $|g(t)| \leq \varepsilon, t \in J$ and $\left|g_{k}\right| \leq \varepsilon, k=1,2, \ldots, m$;

(ii) $y^{\prime}(t)=f(t, y(t))+g(t), t \in J^{\prime}$

(iii) $\Delta y\left(t_{k}\right)=I_{k}\left(y\left(t_{k}^{-}\right)\right)+g_{k}, k=1,2, \ldots, m$.

Proposition 2 If $y \in P C^{1}(J, \mathbb{R})$ is a solution of inequality (8), then $y$ is a solution of the following inequality

$$
\left|y(t)-u_{T}+\sum_{p=0}^{k-1} I_{m-p}\left(y\left(t_{m-p}^{-}\right)\right)+\int_{t}^{T} f(s, y(s)) d s\right| \leq(m+t-T) \varepsilon
$$

for all $t \in J^{\prime}$.

Proof. Indeed, by proposition 1, we have

$$
\left\{\begin{array}{l}
y^{\prime}(t)=f(t, y(t))+g(t), \quad t \in J^{\prime} \\
\Delta y\left(t_{k}\right)=I_{k}\left(y\left(t_{k}^{-}\right)\right)+g_{k}, \quad k=1,2, \ldots, m .
\end{array}\right.
$$

Then, for $t \in\left(t_{m-k}, t_{m-k+1}\right)$ and $k=0, \ldots, m$,

$$
\begin{aligned}
y(t)= & u_{T}-\sum_{p=0}^{k-1} I_{m-p}\left(y\left(t_{m-p}^{-}\right)\right)-\sum_{p=0}^{k-1} g_{i} \\
& -\int_{t}^{T} f(s, y(s)) d s-\int_{t}^{T} g(s) d s .
\end{aligned}
$$

From here it follows that

$$
\begin{aligned}
& \left|y(t)-u_{T}+\sum_{p=0}^{k-1} I_{m-p}\left(y\left(t_{m-p}^{-}\right)\right)+\int_{t}^{T} f(s, y(s)) d s\right| \\
\leq & \sum_{i=1}^{m}\left|g_{i}\right|+\int_{t}^{T}|g(s)| d s \leq m \varepsilon+\varepsilon \int_{T}^{t} d s \\
\leq & m \varepsilon-\varepsilon T+\varepsilon t=(m+t-T) \varepsilon .
\end{aligned}
$$

Similar remarks or propositions hold true for the solutions of the inequalities (9) and (10).

Note that the Ulam stabilities of the impulsive differential equations are some special types of data dependence of the solutions of impulsive differential equations. 
Theorem 10 Let the assumptions (H1), (H2) and (H5) hold and suppose there exists $\lambda_{\varphi}>0$ such that

$$
\int_{t}^{T} \varphi(s) d s \leq \lambda_{\varphi} \varphi(t)
$$

for each $t \in J$, where $\varphi \in P C^{1}\left(J, \mathbb{R}^{+}\right)$is nondecreasing. Then the equation (1) is generalized Ulam-Hyers-Rassias stable with respect to $(\varphi, \psi)$.

Proof. Let $y \in P C^{1}(J, \mathbb{R})$ be a solution to the inequality (9). Denote by $u$ the unique solution of the backward impulsive problem

$$
\begin{cases}u^{\prime}(t)=f(t, u(t)), & t \in J^{\prime}=[0, T], t \neq t_{k} \\ \left.\Delta u\right|_{t=t_{k}}=I_{k}\left(u\left(t_{k}^{-}\right)\right), & k=1, \ldots, m \\ u(T)=u_{T} & \end{cases}
$$

Then we have

$$
u(t)=u_{T}-\sum_{p=0}^{k-1} I_{m-p}\left(u\left(t_{m-p}^{-}\right)\right)-\int_{t}^{T} f(s, u(s)) d s,
$$

where $t \in\left(t_{m-k}, t_{m-k+1}\right)$ for $k=0, \ldots, m$.

Differentiating the inequality (9) (see Proposition 2), for each $t$ in $\left(t_{m-k}, t_{m-k+1}\right)$, we obtain

$$
\begin{aligned}
\mid y(t) & -u_{T}+\sum_{p=0}^{k-1} I_{m-p}\left(y\left(t_{m-p}^{-}\right)\right)+\int_{t}^{T} f(s, y(s)) d s \mid \\
& \leq \sum_{i=1}^{m}\left|g_{i}\right|+\int_{t}^{T} \varphi(s) d s \\
& \leq m \psi+\lambda_{\varphi} \varphi(t) \leq(\varphi(t)+\psi)\left(\lambda_{\varphi}+m\right) .
\end{aligned}
$$

Hence, for each $t \in\left(t_{m-k}, t_{m-k+1}\right)$ and $k=0, \ldots, m$, we can write

$$
\begin{aligned}
|y(t)-u(t)| \leq & \left|y(t)-u_{T}+\sum_{p=0}^{k-1} I_{m-p}\left(y\left(t_{m-p}^{-}\right)\right)+\int_{t}^{T} f(s, y(s)) d s\right| \\
& +\sum_{p=0}^{k-1}\left|I_{m-p}\left(y\left(t_{m-p}^{-}\right)\right)-I_{m-p}\left(u\left(t_{m-p}^{-}\right)\right)\right| \\
& +\int_{t}^{T}|f(s, y(s))-f(s, u(s))| d s \\
\leq & (\varphi(t)+\psi)\left(\lambda_{\varphi}+m\right)+\sum_{p=0}^{k-1} \mu_{k}\left|\left(y\left(t_{m-p}^{-}\right)\right)-\left(u\left(t_{m-p}^{-}\right)\right)\right| \\
& +\lambda \int_{t}^{T}|y(s)-u(s)| d s .
\end{aligned}
$$


Finally, by Lemma 1, we obtain

$$
|y(t)-u(t)| \leq(\varphi(t)+\psi)\left(\lambda_{\varphi}+m\right) \prod_{t<t_{k}<T}\left(1+\mu_{k}\right) \exp (\lambda(T-t)) .
$$

Thus, equation (1) is generalized Ulam-Hyers-Rassias stable with respect to $(\varphi, \psi)$. The proof is completed.

Remark 2 Using the approach developed in [24], one can prove the validity of the following statements.

1. Under the assumptions of Theorem 10, if we consider the equation (1) and inequality (8), by the same process we can verify that the equation (1) is Ulam-Hyers stable.

2. Under the assumptions of Theorem 10, if we consider the equation (1) and inequality 10 , we can use the same process to verify that the equation (1) is Ulam-Hyers-Rassias stable with respect to $(\varphi, \psi)$.

3. The above results can be extended to the case of the equation (2).

\section{$6 \quad$ Examples}

\section{Example 1}

Consider the backward impulsive ordinary differential equation

$$
\left\{\begin{array}{l}
u^{\prime}(t)=0, \quad t \in J^{\prime}=[0,1]-\{1 / 2\} \\
\left.\Delta u\right|_{t=\frac{1}{2}}=\left|u\left(\frac{1}{2}^{-}\right)\right|\left(1+\left|u\left(\frac{1}{2}^{-}\right)\right|\right)^{-1} \\
u(1)=1
\end{array}\right.
$$

and the inequalities

$$
\left\{\begin{array}{l}
\left|y^{\prime}(t)\right| \leq \varepsilon, \quad t \in J^{\prime}=[0,1]-\{1 / 2\} \\
|\Delta y|_{t=\frac{1}{2}}-\left|y\left(\frac{1}{2}^{-}\right)\right|\left(1+\left|y\left(\frac{1}{2}^{-}\right)\right|\right)^{-1} \mid \leq \varepsilon, \quad \varepsilon>0 .
\end{array}\right.
$$

Let $y \in P C^{1}([0,1], \mathbb{R})$ be a solution to the inequality $(12)$. Then there exist $g \in P C^{1}([0,1], \mathbb{R})$ and $g_{1} \in \mathbb{R}$ such that

$$
\begin{gathered}
|g(t)| \leq \varepsilon, \quad t \in[0,1], \\
y^{\prime}(t)=g(t), \quad t \in J^{\prime}=[0,1]-\{1 / 2\}, \\
\left.\Delta y\right|_{t=\frac{1}{2}}=\left|y\left(\frac{1^{-}}{2}\right)\right|\left(1+\left|y\left(\frac{1^{-}}{2}\right)\right|\right)^{-1}+g_{1} .
\end{gathered}
$$


Integrating (13) from $t$ to 1 via (14), we obtain

$$
\begin{aligned}
y(t) & =y(1)-\left(I_{\frac{1}{2}}\left(y\left(t_{\frac{1}{2}}^{-}\right)\right)+g_{1}\right)-\int_{t}^{1} g(s) d s \\
& =y(1)-\left(\frac{\left|y\left(\frac{1}{2}^{-}\right)\right|}{1+\left|y\left(\frac{1}{2}^{-}\right)\right|}+g_{1}\right)-\int_{t}^{1} g(s) d s .
\end{aligned}
$$

Let us consider the solution $u$ of 11 given by

$$
u(t)=y(1)-\left|u\left(\frac{1}{2}^{-}\right)\right|\left(1+\left|u\left(\frac{1}{2}^{-}\right)\right|\right)^{-1} .
$$

Then we can write

$$
\begin{aligned}
|y(t)-u(t)| & =\left|\frac{\left|u\left(\frac{1}{2}^{-}\right)\right|}{1+\left|u\left(\frac{1}{2}^{-}\right)\right|}-\frac{\left|y\left(\frac{1}{2}^{-}\right)\right|}{1+\left|y\left(\frac{1}{2}^{-}\right)\right|}-g_{1}-\int_{t}^{1} g(s) d s\right| \\
& \leq\left|\frac{\left|u\left(\frac{1}{2}^{-}\right)\right|}{1+\left|u\left(\frac{1}{2}^{-}\right)\right|}-\frac{|y|}{1+\left|y\left(\frac{1}{2}^{-}\right)\right|}\right|+\left|g_{1}\right|+\left|\int_{t}^{1} g(s) d s\right| \\
& \leq|| u\left(\frac{1^{-}}{2}\right)|-| y\left(\frac{1^{-}}{2}\right)||+\left|g_{1}\right|+\left|\int_{t}^{1} g(s) d s\right| \\
& \leq\left|u\left(\frac{1^{-}}{2}\right)-y\left(\frac{1^{-}}{2}\right)\right|+\left|g_{1}\right|+\int_{t}^{1}|g(s)| d s \\
& \leq\left|u\left(\frac{1^{-}}{2}\right)-y\left(\frac{1^{-}}{2}\right)\right|+\varepsilon+\varepsilon \int_{t}^{1} d s, \quad t \in[0,1] \\
& \leq\left|u\left(\frac{1^{-}}{2}\right)-y\left(\frac{1^{-}}{2}\right)\right|+\varepsilon+\varepsilon(1-t), \quad t \in[0,1] \\
& \leq 3 \varepsilon+\varepsilon(1-t)=\varepsilon(4-t), \quad t \in[0,1] .
\end{aligned}
$$

Thus, Equation (11) is generalized Ulam-Hyers stable, which is a special case of generalized Ulam-Hyers-Rassias stable.

\section{Example 2}

Consider the backward impulsive ordinary differential equation

$$
\begin{cases}u^{\prime}(t)=\frac{1}{5+e^{t}+u^{2}(t)}, & t \in J^{\prime}=[0, \pi], t \neq t_{k}, \\ \left.\Delta u\right|_{t=t_{k}}=\frac{1}{5 k+u^{2}\left(t_{k}^{-}\right)}, & k=1, \ldots, 5, t_{k}=k \frac{\pi}{5}, \\ u(\pi)=1 .\end{cases}
$$


Set

$$
f(t, x)=\frac{1}{5+e^{t}+x^{2}}, \quad(t, x) \in J \times[0,+\infty),
$$

and

$$
I_{k}(x)=\frac{1}{5 k+x^{2}} .
$$

Let $(x, y) \in[0,+\infty)$ and $t \in J$. By the mean value theorem, we have

$$
\begin{aligned}
|f(t, x)-f(t, y)| & =\frac{2 \xi}{\left(5+e^{t}+\xi^{2}\right)^{2}}|x-y| \\
& \leq \frac{2 \xi}{\left(6+\xi^{2}\right)^{2}}|x-y|, \quad x<\xi<y .
\end{aligned}
$$

Then

$$
|f(t, x)-f(t, y)| \leq \frac{\sqrt{2}}{32}|x-y|
$$

Hence, the condition (H2) holds with $\lambda=\sqrt{2} / 32$.

Let $(x, y) \in[0,+\infty)$. By the mean value theorem, we have

$$
\left|I_{k}(x)-I_{k}(y)\right|=\frac{2 \xi}{\left(5+\xi^{2}\right)^{2}}|x-y|, \quad x<\xi<y
$$

Then

$$
\left|I_{k}(x)-I_{k}(y)\right| \leq \frac{3 \sqrt{15}}{200}|x-y|
$$

Hence, the condition (H3) holds with $\mu=3 \sqrt{15} / 200$.

Since

$$
5 \times \frac{3 \sqrt{15}}{200}+\frac{\pi \sqrt{2}}{32}=\frac{3 \sqrt{15}}{50}+\frac{\pi \sqrt{2}}{32}=\frac{48 \sqrt{15}+25 \pi \sqrt{2}}{800}<1 .
$$

the condition (5) is satisfied. Thus, by Theorem 6, the problem (15) has a unique solution.

Now, we proceed as in the proof of Theorem 10. Let $u \in P C^{1}(J, \mathbb{R})$ be a solution of the inequality (9). Denote by $x$ the unique solution to the backward impulsive problem (15). Then we obtain

$$
u(t)=1-\sum_{p=0}^{k-1} I_{5-p}\left(u\left(t_{5-p}^{-}\right)\right)-\int_{t}^{\pi} \frac{d s}{5+e^{s}+u^{2}(s)},
$$

where $t \in\left(t_{5-k}, t_{5-k+1}\right)$ for $k=0, \ldots, 5$. 
Like in Proposition 2, by differentiation of the inequality, for each $t \in\left(t_{5-k}, t_{5-k+1}\right)$, we have

$$
\begin{aligned}
\mid u(t) & -1+\sum_{p=0}^{k-1} I_{5-p}\left(u\left(t_{5-p}^{-}\right)\right)+\int_{t}^{\pi} \frac{d s}{5+e^{s}+u^{2}(s)} \mid \\
& \leq \sum_{i=1}^{5}\left|g_{i}\right|+\int_{t}^{\pi} \varphi(s) d s \\
& \leq 5 \psi+\lambda_{\varphi} \varphi(t) \\
& \leq(\varphi(t)+\psi)\left(\lambda_{\varphi}+3\right) .
\end{aligned}
$$

Hence, for each $t \in\left(t_{5-k}, t_{5-k+1}\right)$ and $k=0, \ldots, 5$, we can write

$$
\begin{aligned}
|u(t)-x(t)| \leq & \left|u(t)-1+\sum_{p=0}^{k-1} I_{5-p}\left(u\left(t_{5-p}^{-}\right)\right)+\int_{t}^{\pi} \frac{d s}{5+e^{s}+u^{2}(s)}\right| \\
& +\sum_{p=0}^{k-1}\left|I_{5-p}\left(u\left(t_{5-p}^{-}\right)\right)-I_{5-p}\left(x\left(t_{5-p}^{-}\right)\right)\right| \\
& +\int_{t}^{\pi}|f(s, u(s))-f(s, x(s))| d s \\
\leq & (\varphi(t)+\psi)\left(\lambda_{\varphi}+5\right)+\sum_{p=0}^{k-1} \mu_{k}\left|\left(u\left(t_{5-p}^{-}\right)\right)-\left(x\left(t_{5-p}^{-}\right)\right)\right| \\
& +\lambda \int_{t}^{\pi}|u(s)-x(s)| d s .
\end{aligned}
$$

By Lemma 1, we obtain

$$
|u(t)-x(t)| \leq(\varphi(t)+\psi)\left(\lambda_{\varphi}+5\right) \prod_{t<t_{k}<T}\left(1+\mu_{k}\right) \exp (\lambda(\pi-t)) .
$$

Recalling that $\lambda=\sqrt{2} / 32$ and $\mu_{k}=3 \sqrt{15} / 200$, we find

$$
\begin{aligned}
|u(t)-x(t)| & \leq(\varphi(t)+\psi)\left(\lambda_{\varphi}+5\right) \prod_{t<t_{k}<T}\left(1+\frac{3 \sqrt{15}}{200}\right) \exp \left(\frac{\sqrt{2}}{32}(\pi-t)\right) \\
& =(\varphi(t)+\psi)\left(\lambda_{\varphi}+5\right)\left(1+\frac{3 \sqrt{15}}{200}\right)^{k} \exp \left(\frac{\sqrt{2}}{32}(\pi-t)\right) \\
& \leq(\varphi(t)+\psi)\left(\lambda_{\varphi}+5\right)\left(1+\frac{3 \sqrt{15}}{200}\right)^{k} e^{\pi-t},
\end{aligned}
$$

$t<t_{k}<\pi$. This implies that the problem (15) is generalized Ulam-HyersRassias stable with respect to $(\varphi, \psi)$. 


\section{Conclusion}

In this paper, we investigate the existence of solutions for backward impulsive differential equations on Banach spaces. The main challenges were overcome by using different classical fixed point theorems to establish the existence of solutions to our problem by adding suitable conditions on the nonlinear term. We succeeded to obtain a unique solution by using the Banach contraction principle and at least one solution by using other fixed point theorems such as Schaefer's and Krasnosel'skii fixed point theorem, both in the cases of local and nonlocal conditions. Further, we obtain generalized-UlamHyers-Rassias stability results for our problem, and we presented examples to illustrate the consistency of our theoretical findings.

It is important to note that there are many applications of backward impulsive differential equations and their stability.

Acknowledgements. The authors would like to express their cordial thanks to the anonymous referee and the editors for their careful reading of the manuscript and their insightful comments, suggestions and the useful remarks, which vastly contributed to improving the presentation of the first version of this paper.

\section{References}

[1] D.D. Bainov and S.G., Integral inequalities of Gronwall type for piecewise continuous functions, J. Appl. Math. Stoch. Anal., 10(1997), pp. 89-94.

[2] D. Bainov and P. Simeonov, Integral inequalities and applications, Kluwer Academic Publishers, Dordrecht, 1992.

[3] D. Bainov and P. Simeonov, Impulsive differential equations, asymptotic properties of the solutions, Series on Advances in Mathematics for Applied Sciences Vol. 28, World Scientific Publishing Co. Pte. Ltd., Singapore, 1995. https://doi.org/10.1142/2413

[4] M. Benchohra and B. A. Slimani, Existence and uniqueness of solutions to impulsive fractional differential equations, Electron. J. Differ. Equ., 2009 (2009), no. 10, pp. 1-11.

[5] L. Byszewski, Theorems about the existence and uniqueness of solutions of a semilinear evolution nonlocal Cauchy problem, J. Math. Anal. Appl., 162 (1991), no. 2, pp. 494-505. https://doi.org/10.1016/0022247x(91)90164-u 
[6] L. Byszewski, Existence and uniqueness of a classical solutions to a functional-differential abstract nonlocal Cauchy problem, J. Math. Appl. Stoch. Anal., 12 (1999), no. 1, pp. 91-97. https://doi.org/10.1155/s1048953399000088

[7] L. Byszewski and V. Lakshmikantham, Theorem about the existence and uniqueness of solutions of a nonlocal Cauchy problem in a Banach space, Appl. Anal., 40 (1991), no. 1, pp. 11-19. https://doi.org/10.1080/00036819008839989

[8] J.H. Chen, C.C. Tisdell and R. Yuan, On the solvability of periodic boundary value problems with impulse, J. Math. Anal. Appl., 331 (2007), no. 2, pp. 902-912. https://doi.org/10.1016/j.jmaa.2006.09.021

[9] P. Chen and Y. Li, Existence and uniqueness of strong solutions for nonlocal evolution equations, Electron. J. Differ. Equ., 2014 (2014), no. 18, pp. 1-9.

[10] J.P. Dauer, N.I. Mahmudov and M.M. Matar, Approximate controllability of backward stochastic evolution equations in Hilbert spaces, J. Math. Anal. Appl., 323 (2006), no. 1, pp. 42-56. https://doi.org/10.1016/j.jmaa.2006.09.021

[11] M. Federson and S. Schwabik, Generalized ODE approach to impulsive retarded functional differential equations, Differ. Integral Equ., 19 (2006), no. 11, pp. 1201-1234.

[12] E. Hernandez and H.R. Henriquez, Impulsive partial neutral differential equations, Appl. Math. Lett., 19 (2006), no. 3, pp. 215-222.

[13] E. Hernandez, H.R. Henriquez and R.Marco, Existence of solutions for a class of impulsive partial neutral functional differential equations, J. Math. Anal. Appl., 331 (2007), no. 2, pp. 1135-1158. https://doi.org/10.1016/j.jmaa.2006.09.043

[14] E. Hernandez, R. Sakthivel and S. Tanaka, Existence results for impulsive evolution differential equations with state-dependent delay, Electron. J. Differ. Equ., 2008 (2008), no. 28, pp. 1-11.

[15] V. Lakshmikantham, D. D. Bainov and P. S. Simeonov, Theory of impulsive differential equations, Worlds Scientific, Singapore, 1989. https://doi.org/10.1142/0906

[16] X.N. Lin and D.Q. Jiang, Multiple positive solutions of Dirichlet boundary value problems for second order impulsive differential equations, J. Math. Anal. Appl., 321 (2006), no. 2, pp. 501-514. https://doi.org/10.1016/j.jmaa.2005.07.076 
[17] R.H. Martin, Nonlinear operators and differential equations in Banach spaces, Robert E. Krieger Publ. Co., Florida, 1987.

[18] M. Matar, Existence of solution to fractional nonlinear backward differential equations on Banach spaces, Int. J. Math. Anal., 6 2012, no. 33, pp. 1641-1647.

[19] J.J. Nieto, Impulsive resonance periodic problems of first order, Appl. Math. Letters, 15 (2002), no. 4, pp. 489-493. https://doi.org/10.1016/s0893-9659(01)00163-x

[20] R. Poongodi and R. Murugesu, Existence of solutions for fractional impulsive integro-differential systems, Malaya J. Mat., S(1) (2013), pp. $56-65$.

[21] A.V. Rezounenko and J. Wu, A non-local PDE model for population dynamics with state-selective delay: Local theory and global attractors, J. Comput. Appl. Math., 190 (2006), no. 1-2, pp. 99-113. https://doi.org/10.1016/j.cam.2005.01.047

[22] Y.V. Rogovchenko, Nonlinear impulse evolution systems and applications to population models, J. Math. Anal. Appl., 207 (1997), no. 2, pp. 300-315. https://doi.org/10.1006/jmaa.1997.5245

[23] A.M. Samoilenko and N.A. Perestyuk, Impulsive differential equations, World Scientific, Singapore, 1995.

[24] J.R. Wang, M. Feckan and Y. Zhou, Ulam's type stability of impulsive ordinary differential equations, J. Math. Anal. Appl., 395 (2012), pp. 258-264.

[25] W. Wei, X. Xiang and Y. Peng, Nonlinear impulsive integro-differential equation of mixed type and optimal controls, Optimization, 55 (2006), no. 1-2, pp. 141-156. https://doi.org/10.1080/02331930500530401

Abdelouahab Mahmoudi

Analysis Department, Faculty of Mathematics,

USTHB-University,

P.O. Box 32 El Alia Bab Ezzouar 16111, Algiers, Algeria.

aomahmoudi@yahoo.fr

Arezki Kessi

Analysis Department, Faculty of Mathematics,

USTHB-University,

P.O. Box 32 El Alia Bab Ezzouar 16111, Algiers, Algeria.

arkessi@yahoo.com 
Please, cite to this paper as published in Armen. J. Math., V. 13, N. 8(2021), pp. 1 21

https://doi.org/10.52737/18291163-2021.13.8-1-21 